\title{
Talent transfer: A systematic review
}

Talent transfer (TT): The process in which athletes make a switch from their original sport (i.e. donor sport) to a new sport (i.e. transfer sport). Previously developed skills are recycled to the transfer sport, so that athletes can fast-track to the top-level.

Aim: To give an overview of studies examining TT.

\section{Findings:}

- TT can serve as an alternative pathway in addition to traditional

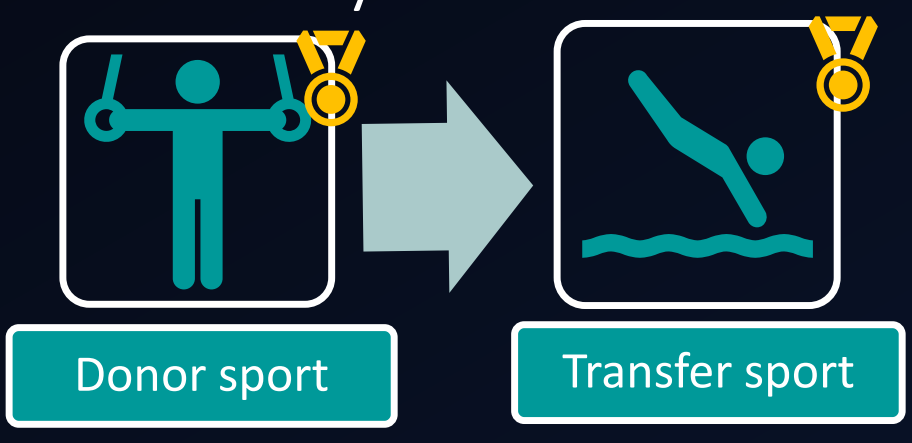
talent development programs.

- Similarities between donor and transfer sports seem helpful but are not a prerequisite.

- Psychological factors (f.e., confidence) play an important role $\&$ athletes might benefit from $\uparrow$ generic athletic ability.

- Knowledge regarding other determining factors of TT is still scarce.

- Athletes, national governing bodies and talent programs might benefit from TT, but empirical evidence for the effectiveness is still lacking. 